Revue musicale OICRM

\title{
Entre pédagogie et médiation : au coeur du partage des pratiques. Entretien avec Jérôme Thiébaux
}

\section{Sophie Renaudin}

Volume 7, numéro 2, 2020

URI : https://id.erudit.org/iderudit/1072420ar

DOI : https://doi.org/10.7202/1072420ar

Aller au sommaire du numéro

Éditeur(s)

Observatoire interdisciplinaire de création et recherche en musique (OICRM)

ISSN

2368-7061 (numérique)

Découvrir la revue

Citer ce document

Renaudin, S. (2020). Entre pédagogie et médiation : au coeur du partage des pratiques. Entretien avec Jérôme Thiébaux. Revue musicale OICRM, 7(2),

125-130. https://doi.org/10.7202/1072420ar
Résumé de l'article

Dans cet entretien, Jérôme Thiébaux, professeur agrégé d'éducation musicale, revient sur son parcours et évoque les différentes expériences qui l'ont conduit à côtoyer, à appréhender, ou encore à pratiquer, la médiation. Il s'agit ici de saisir les diverses formes - mais aussi les différents noms - que revêt la médiation, tout en réfléchissant à ce qui fait sa spécificité. Les réflexions menées par Jérôme Thiébaux nous permettent alors d'envisager la posture adoptée par celle ou celui qui pratique la médiation dans différents domaines, tant dans celui des actions culturelles des orchestres que dans celui de l'enseignement. Son point de vue original nous conduit ainsi à comprendre les rapports qui lient médiation et pédagogie et à repenser les relations qui se nouent au sein de la salle de classe. 


\title{
Entre pédagogie et médiation : au coeur du partage des pratiques. Entretien avec Jérôme Thiébaux \\ Sophie Renaudin
}

\begin{abstract}
Résumé
Dans cet entretien, Jérôme Thiébaux, professeur agrégé d'éducation musicale, revient sur son parcours et évoque les différentes expériences qui l'ont conduit à côtoyer, à appréhender, ou encore à pratiquer, la médiation. Il s'agit ici de saisir les diverses formes - mais aussi les différents noms - que revêt la médiation, tout en réfléchissant à ce qui fait sa spécificité. Les réflexions menées par Jérôme Thiébaux nous permettent alors d'envisager la posture adoptée par celle ou celui qui pratique la médiation dans différents domaines, tant dans celui des actions culturelles des orchestres que dans celui de l'enseignement. Son point de vue original nous conduit ainsi à comprendre les rapports qui lient médiation et pédagogie et à repenser les relations qui se nouent au sein de la salle de classe.
\end{abstract}

Mots clés : école ; médiation culturelle ; pédagogie ; pratique ; Jérôme Thiébaux.

\begin{abstract}
In this interview, Jérôme Thiébaux, professor of music education, looks back over his career and discusses the different experiences that led him to come into contact with, understand and practice mediation. This article is an opportunity to grasp the various forms - but also the different names - that mediation takes on, while reflecting on what makes it special. Jérôme Thiébaux's thoughts allow us to consider the position adopted by those who practice mediation in different fields, both in the educational activities of orchestras and in teaching. His original point of view thus leads us to understand the relationships between mediation and pedagogy and to rethink the relationships that are established within the classroom.
\end{abstract}

Keywords: cultural mediation; pedagogy; practice; school; Jérôme Thiébaux. 
Agrégé de musique et professeur d'éducation musicale, Jérôme Thiébaux œuvre depuis longtemps dans le domaine de la médiation de la musique. Pendant dix ans, il a été responsable des actions culturelles et pédagogiques de l'Orchestre national de Lyon (2000-2010), avant de prendre la direction administrative et générale de 1'Orchestre Victor Hugo de Franche-Comté. Formé au cNsm (Conservatoire national et supérieur de musique) dans les classes de hautbois et d'histoire de la musique, Thiébaux considère, dès ses premières années de formation, que tout le monde devrait pouvoir bénéficier de la circulation des savoirs et des pratiques qui a lieu au sein de cet établissement. Pour lui, la médiation est une question de posture et d'attitude : pour être médiateur, il faut se donner les moyens d'être disponible, de s'ouvrir aux autres comme individu ou comme institution culturelle.

\section{UNE THÉORISATION DE LA MÉDIATION NÉE DE LA PRATIQUE}

Jérôme Thiébaux : Ma définition de la médiation serait en rapport avec ce que je définirais comme "ma pratique ». Je pense, en effet, que c'est au fur et à mesure de pratiques, que je n'ai pas toujours définies comme de la médiation, que je suis arrivé au terme " médiation ". J'ai aussi lu et rencontré plusieurs personnes qui réfléchissaient à ces questions. Je ne sais pas vraiment comment, moi, je définirais la médiation; ce n'est peut-être pas nécessairement à moi de la définir. Par contre, je peux trouver dans différents textes théoriques, ou dans certains témoignages, une définition qui entre en écho avec ma pratique. C'est de cette manière que s'est enclenchée l'idée que je faisais de la médiation. Quand j'ai commencé ma carrière, ce terme-là était très peu utilisé dans le monde de la musique. On parlait des " actions culturelles », de la « pédagogie »... C'est aussi en côtoyant le monde des musées, les collègues du Musée des Beaux-Arts de Lyon par exemple, qui, eux, s'appelaient

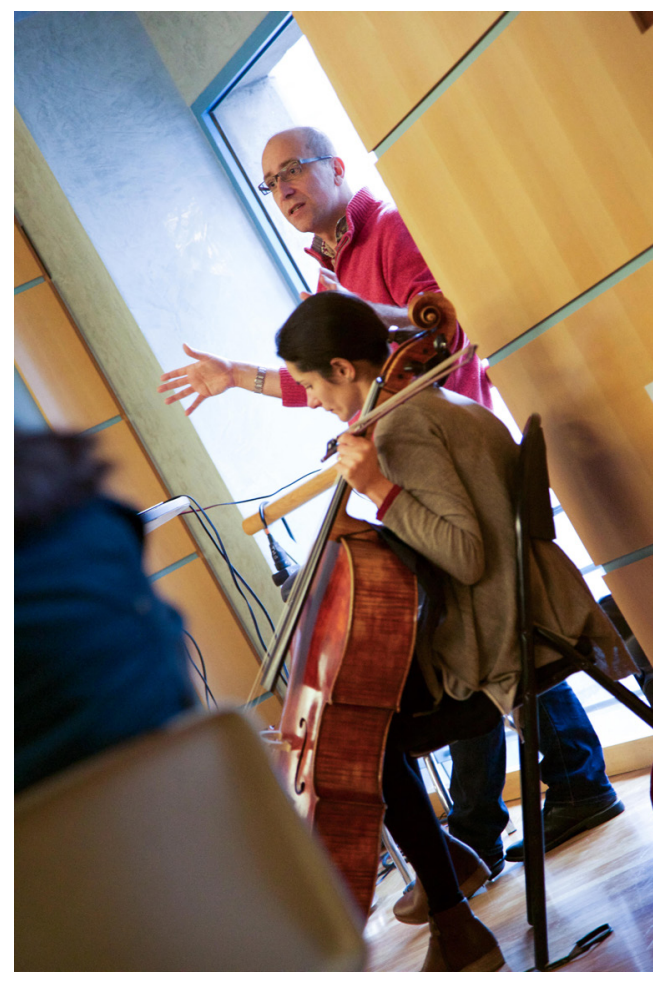

Figure 1 : Jérôme Thiébaux lors des rencontres "Pause dej" organisées avec des musiciens de l'Orchestre Victor Hugo Franche-Comté. Photo : Chloé Stiefvater, 2016. " médiateurs ». En travaillant et en développant des projets avec eux, le terme a commencé à résonner. Je me suis dit : "Tiens, au-delà de l'action culturelle, de l'action artistique et pédagogique, est-ce qu'il n'y a pas quelque chose d'autre qui va venir chapeauter tout cela ? » Il s'agissait de trouver une cohérence à des choses très éparses. C'est de là que peut venir ma propre définition. La médiation est une sorte de posture : qu'est-ce que l'on est en tant qu'acteurs quand on est dans un projet, de type $x, y, z$ ? Quelle est notre propre attitude ? De quelle manière souhaite-t-on se projeter vers un public? Quelle est la manière dont on pense le projet? Je pense que le terme 
" médiation » a beaucoup servi à inventer, porter ou même expliquer des projets, à se dire que l'on est des « passeurs » de quelque chose...

Sophie Renaudin : Vous avez mentionné le fait que le terme "médiation "s'est d'abord imposé dans le domaine des musées. Pensez-vous qu'il existe une différence de posture entre médiation culturelle et médiation de la musique?

J. т. : Si l'on a envie de donner le goût, de découvrir, de partager, sous forme d'expériences... alors il n'y a pas nécessairement de frontières entre la médiation dans un musée ou la médiation de la musique. Mais la matière, elle, diffère, ainsi que le rapport de la société à l'objet. Il y a une forme de mythologie autour de la musique, c'est sans doute pour cela que je me suis emparé du mot " médiation »... Il faut dépasser l'idée d'une musique hermétique, destinée à des spécialistes.

Je dirais qu'entre médiation culturelle et médiation de la musique, les dispositifs changent, mais la posture mentale et intellectuelle, le principe de mettre en ouvre quelque chose par la parole ou par un dispositif, restent identiques. On a bien fait de s'inspirer les uns les autres !

\section{LA MÉDIATION À PARTIR DE, OU AU SEIN DE, L'ENSEIGNEMENT}

C'est en suivant la voie de l'enseignement que Jérôme Thiébaux s'est rapproché de la posture de médiateur. En arrivant dans le milieu de l'enseignement secondaire, sa formation est celle d'un musicien très spécialisé issu des classes de théorie et de pratique de la musique d'établissements prestigieux. Pour lui, l'enseignement au collège demande nécessairement de faire appel à des outils de médiation. La médiation n'est pas théorisée, elle est immédiatement mise en œuvre. Elle procède d'une volonté de partage de savoirs entre les protagonistes de la salle de classe.

J. т. : Ce que j'apprécie dans l'enseignement, c'est ce partage, un partage réciproque entre le professeur et l'élève. En tant qu'enseignants, on a des programmes imposés, mais on s'en sert : par la musique, on va devenir médiateur de plein d'autres choses. Ce qui m'intéresse dans la médiation, c'est de porter les personnes, en l'occurrence les adolescents, de les conforter, pour qu'ils s'approprient leur propre univers. Il s'agit aussi de les ouvrir à d'autres mondes. Étonnement, en classe, je ne parle jamais de la culture dite "standard ", mais on y revient toujours. On part toujours d'univers qui peuvent être très éloignés des élèves, mais ce qui m'intéresse, c'est de pouvoir faire des ponts avec le leur, avec ce qu'ils entendent.

s. R. : Sous quelle forme se déroule votre enseignement dans les classes de collège et de lycée (ateliers, cours magistraux, etc.)?

J. T. : Dans la classe, les élèves sont toujours en action dans leur créativité. On invente toujours et ils utilisent ce qu'ils sont pour créer. Puis, généralement en petits groupes, on découvre des choses qui entrent en relation avec eux et qui vont résonner avec ce qu'ils sont. Quand on écoute, on est aussi dans l'action, les élèves sont sollicités par rapport à ce qu'ils ressentent, ils s'interrogent sur la manière dont ils reçoivent personnellement la musique. On a trop souvent dit qu'il ne fallait pas parler de musique, ou, du moins, en parler d'une certaine manière. Mais je pense que le fait de parler 
de quelque chose permet de se l'approprier. Même si l'on ne l'aime pas. Si un élève me dit qu'il n'aime pas quelque chose, il a une raison, et, souvent, c'est une bonne raison (s'il me dit, par exemple : "j'ai l'impression que le compositeur veut nous déstabiliser »). C'est à partir de là que l'on crée le dialogue.

Dans nos métiers, on craint souvent d'utiliser un langage très spécifique sur la musique. Ce vocabulaire, on l'utilise quand on veut aller plus loin, mais dans les autres cas, on le met de côté. Cela ne signifie pas que l'on arrive à quelque chose de superficiel, au contraire. Derrière chaque chose que l'on entend, il y a une réception personnelle, et je pense que c'est important de la verbaliser. Par la parole, par le dialogue, on arrive à l'appropriation de ce que l'on entend. Dans le cadre de l'enseignement, c'est très important d'être à l'écoute de ce que les adolescents disent, de ce qu'ils construisent comme idées, qu'elles soient considérées comme "non-savantes » ou déstabilisantes.

S. R. : Vous êtes enseignant, musicien et médiateur même si vous n'utilisez pas ce terme... Accepteriez-vous, et revendiqueriez-vous, le terme de «créateur »?

J. T. : Je ne me suis jamais posé la question ! (Rires.) Je n'ai jamais eu le besoin de m'approprier cette question-là. Même le terme " médiateur ", je ne l'utilise pas nécessairement comme tel, ce n'est pas un mot facile à utiliser. Il est polysémique, et il n'est pas entendu comme un métier particulier, sauf quand on est dans le domaine juridique. Il y a de véritables difficultés à cerner l'objet ; c'est plus facile de dire : " je suis prof » ou " je suis responsable d'un service d'actions culturelles ». Pour le terme "créateur », oui, c'est une possibilité de dire que l'on crée dans un domaine artistique, mais, pour moi, ce n'est pas nécessairement une préoccupation d'avoir à me positionner par rapport à cette question... Ça viendra peut-être !

Mais je comprends mes collègues qui sont dans des démarches ou des dispositifs dans lesquels il y a un fort travail personnel, un travail d'écriture ou de fiction par exemple, ou plus généralement un travail dans le domaine musical, littéraire ou plastique. Il y a un besoin de les reconnaître comme des créateurs, ou des inventeurs, et pas simplement comme des personnes qui se situeraient dans une sous-catégorie de ces termes.

\section{LA MUSIQUE (ET LA MÉDIATION) POUR TOUS}

À côté de son métier d'enseignant, Jérôme Thiébaux anime des rencontres dans un lieu associatif à Besançon. Pour ces ateliers, l'idée est de partager les savoirs et les pratiques de chacun, en mettant en place une égalité de principe entre tous les participants. Apparaît ainsi une certaine forme de médiation, même si, encore une fois, elle ne prend pas toujours ce nom-là.

J. T. : Depuis que je suis arrivé à Besançon, je me suis engagé, à titre personnel, pour des ateliers d'écoute de la musique. On se réunit un samedi par mois et on écoute ensemble de la musique, une musique que j'ai généralement choisie au hasard, enfin, presque. C'est gratuit et ouvert à tous. Dans l'assemblée, il y a des gens qui écoutent et qui ont envie de partager... Ce qui m'intéresse, c'est qu'ils ont toujours quelque chose à dire. On développe cette parole et cette écoute et moi je m'exprime sur ce que j'entends aussi, en allant plus loin parce que dans l'assemblée, je suis peut-être celui 
qui a eu la chance de lire plus de biographies de Bach, mais c'est tout. Tout le monde apporte son savoir, c'est un moment de partage et de découverte. L'atelier a lieu au Café des pratiques à Besançon. C'est un lieu associatif où les gens se rencontrent, échangent leurs pratiques, et les ateliers se déroulent dans cette optique : celui qui aime sa pratique, qui a quelque chose à faire partager, le fait découvrir aux autres. Dans ce cadre, je ne suis pas nécessairement présenté avec un titre, je ne suis pas médiateur, je ne suis pas professeur, je suis juste la personne qui propose ça.

S. R. : Vous avez donc travaillé avec des associations, des écoles, des orchestres... Quand on fait de la médiation, avec quels partenaires doit-on travailler?

J. T. : Tout le monde doit pouvoir être partenaire. Évidemment, chaque partenaire a ses avantages et ses inconvénients. L'institution peut paraître contraignante ${ }^{1}$, mais elle a les moyens ; il existe un véritable confort lié à cette position. Néanmoins, quand j'ai commencé au sein d'une institution, il fallait convaincre. Encore aujourd'hui, l'institution doit être convaincue du bien-fondé de la cause. Et là encore, on a peut-être de la médiation à faire... À l'inverse, quand on est dans une association, on peut tous être convaincus du bien-fondé de la cause, mais il va falloir expliquer, se battre pour trouver des financements, par exemple. C'est un métier militant dans une certaine mesure.

Ce qui est simple, c'est quand les gens ont envie de le faire. Ce que j'aime avec l'école, c'est que l'on touche tout le monde. Tous les enfants ont le droit. J'ai toujours été partisan de cette approche assez large, pouvoir aller au concert ou chercher les moyens de s'y rendre...

S. R. : Votre type idéal d'activité de médiation ?

J. T. : Je ne pense pas qu'il existe une forme de médiation idéale en soi. Parfois, on se retrouve dans des situations non prévues, qui semblaient, certes, idéales, mais qui ne marchent pas. Il n'y a pas de typologie. La question est plutôt de savoir là où le public est le plus à l'aise.

La difficulté dans la médiation de la musique est que l'on a du mal à mettre en action des publics que l'on considère non musiciens. Pourtant, on peut les considérer comme musiciens dans l'action. Cela demande d'être convaincu du fait qu'une personne peut jouer de la musique à partir du moment où elle organise deux sons, même si ces sons ne sont, soi-disant, pas "justes ». Je considère que tout le monde peut, à partir de trois minutes d'expérience, se dire "musicien ". C'est cette idée que j'essaye de transmettre, à la fois en tant que praticien et en tant qu'écoutant, au Café des pratiques à Besançon. On a à travailler l'idée que tout le monde écoute. Notre différence avec quelqu'un qui écoute mais qui, soi-disant, n'est pas musicien, c'est notre vocabulaire. Mais, après tout, en tant que musiciens, on se trouve tout autant démunis dans une autre situation...

1 Jérôme Thiébaux cite l'essai de Serge Saada (Et si l'on partageait la culture ? Essai sur la médiation culturelle et le potentiel du spectateur, Toulouse, Éditions de l'Attribut, 2011) pour souligner les difficultés et les contraintes liées au fonctionnement de l'institution. 
S. R. : Avez-vous des projets à venir?

J. T. : Un jour, je pense que j'ouvrirai une école de musique... Pour que, justement, tout le monde puisse pratiquer et que tout le monde puisse profiter de ce que les autres font. Tout le monde devrait pouvoir accéder à ce qui est le bien commun, c'est-à-dire au théâtre, à une salle de concert... Et ça, ça passe aussi par l'idée d'aller voir les gens, de les rassurer, de leur dire qu'ils sont plus importants que ce qu'ils vont voir. Il y a également beaucoup de personnes qui ont envie de faire de la musique mais qui ne le peuvent pas pour plusieurs raisons. Par exemple, les Conservatoires leur sont fermés. Je pense que l'on est trop exigeant dans nos apprentissages de performance et d'excellence. On devrait pouvoir aller prendre deux cours de guitare, s'en satisfaire, et revenir trois mois après ! Ça m'amuserait bien. Ce qui m'intéresse, c'est que tout le monde puisse s'exprimer, que ce soit par la musique, le théâtre... On a ce besoin fondamental dans notre société : que chacun s'empare de ce qui l'anime. 